Copyright (C) 2006 IEEE. Reprinted from IEEE Transactions on Power Delivery, 2004; 19 (3) :1085-1090

This material is posted here with permission of the IEEE. Such permission of the IEEE does not in any way imply IEEE endorsement of any of the University of Adelaide's products or services. Internal or personal use of this material is permitted. However, permission to reprint/republish this material for advertising or promotional purposes or for creating new collective works for resale or redistribution must be obtained from the IEEE by writing to pubs-permissions@ieee.org.

By choosing to view this document, you agree to all provisions of the copyright laws protecting it. 


\title{
Identification of the Resonant-Grounded System Parameters by Evaluating Fault Measurement Records
}

\author{
Rastko Živanović, Member, IEEE, Peter Schegner, Member, IEEE, Olaf Seifert, and Georg Pilz
}

\begin{abstract}
The operation of a resonant-grounded network during an earth-fault condition depends on the three basic parameters: damping, detuning, and unbalance factor. These parameters are influenced by the environmental conditions (e.g. humidity, temperature, and pollution), and the network topology. Accurate values of these parameters during an earth-fault condition are required to examine the operation of the compensation system. The fault records could be used for that purpose. The recorded neutral-to-ground voltage signals have been parameterized (using damping and detuning as parameters) according to the mathematical model of the transient process. Iteratively reweighted least squares algorithm has been used to fit the model. This algorithm is the major improvement over the classical least squares approach. It is able to filter out noise more efficiently. As a direct result, very accurate parameter identification has been achieved. This paper concludes with the practical examples.
\end{abstract}

Index Terms-Digital measurements, grounding, least squares methods, power distribution faults.

\section{INTRODUCTION}

$\mathbf{T}$ HE MOST important advantage of the arc-suppression coil compensated system is the automatic arc extinction in the case of an earth fault condition. Petersen invented the principle of the system in 1919 [1]: the inductive current due to a coil connected between neutral and ground neutralizes the capacitive earth-fault current so that the current flowing through the fault is of insufficient value to support an arc. In order to describe and quantify the phenomena observed in the compensated system during the fault and in unfaulty operation, the three parameters have been used widely [2], [3]. Namely, the parameters are dumping, detuning (also called mismatch), and unbalance factor. Value of the unbalance factor depends on the type of distribution lines: transposed overhead, untransposed overhead, or cable. Also, the capacitive coupling with neighboring lines can influence this parameter. In normal operation, the neutral-to-ground voltage could be prohibitively large for large unbalance factor. To reduce the neutral voltage, the inductance of the coil should be adjusted. This will make detuning parameter different then zero and it will reduce compensation capability of the system. The better solution is to apply transposition on overhead lines. To achieve optimal adjustment of the system during network

Manuscript received May 28, 2003. This work was supported by the German Academic Exchange Services (DAAD).

R. Živanović is with the Department of Power Engineering, Tshwane University of Technology, Pretoria 0001, South Africa (e-mail: zivanovr@ yahoo.com).

P. Schegner, O. Seifert, and G. Pilz are with the Institute of Electrical Power Systems and High Voltage Engineering, Dresden University of Technology, Dresden 01062, Germany (e-mail: schegner@eev.et.tu-dresden.de).

Digital Object Identifier 10.1109/TPWRD.2004.829945

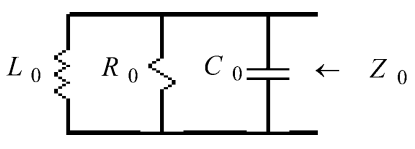

Fig. 1. Zero-sequence circuit representing the earth-fault condition in a resonant-grounded network.

topology changes, various methods for continuous tracking of the basic parameters have been applied [4]. These methods are using the neutral voltage measurement recorded during some perturbation introduced in the system by either a small change of coil inductance or by injecting current into the neutral. The parameters are calculated from either the resonance curve or the loci curve of the neutral voltage [5].

The fault records could be used to analyze and verify the operation of the compensation system. The most suitable recorded waveform is the ringdown of the neutral voltage after an arc is extinguished. In this waveform, the arc nonlinearity does not distort the signal and the level of harmonics is lower. The neutral and phase voltages recover to their prefault values; therefore, we call this transient process the recovery process. The main components of the waveform are two sinusoids with very similar frequencies. These are the power system fundamental frequency and the resonant frequency of the zero-sequence parallel $R L C$ circuit. To estimate parameters of these close frequencies, the high-resolution methods are required [6], [7].

In Section II of this paper, the basic parameters of the resonant-earthed system are described. Section III presents the parameter identification technique. The resonant frequency component of the signal is parameterized using mathematical model of the transient recovery process. To fit the model, the iteratively reweighted least squares (IRLS) method has been used. In the same section, the sources of harmonics in the signal are identified and the use of a low-pass filter has been suggested. The practical results are presented in Section IV.

\section{PARAmeters of the Resonant EARThed System}

The earth-fault condition in a resonant earthed network could be analyzed using the parallel $R L C$ zero-sequence circuit (Fig. 1). All series elements are omitted in the circuit because their values are negligibly small compared to the shunt values. The equivalent zero-sequence impedance representing the circuit in Fig. 1 is equal to

$$
\underline{Z}_{0}=\frac{1}{\frac{1}{R_{0}}+j\left(\omega C_{0}-\frac{1}{\omega L_{0}}\right)}
$$

where $R_{0}$ is the Petersen coil resistance (multiplied by three) in parallel with the zero-sequence network conductance and fault 
resistance. $C_{0}$ is the network zero-sequence capacitance, $L_{0}$ is equal to the Petersen coil inductance multiplied by three, and $\omega$ is the angular fundamental frequency. Underline indicates the complex value.

The earth-fault current could be calculated as

$$
\underline{I}_{e}=\frac{I_{c}}{\omega C_{0} \underline{Z}_{0}}
$$

where $I_{c}$ represents the earth-fault capacitive current without compensation, (i.e. in the case of isolated neutral). By incorporating (1) into (2), we obtained the following expression that describes the earth-fault current $\underline{I}_{e}$ using the earth-fault capacitive current of the isolated system

$$
\underline{I}_{e}=(d+j \nu) I_{c}
$$

where

$$
d=\frac{1}{\omega R_{0} C_{0}}
$$

and

$$
\nu=\left(1-\frac{\omega_{0}^{2}}{\omega^{2}}\right)
$$

are damping and detuning parameters, respectively. Detuning $\nu$ depends on the ideal resonant frequency (energy losses neglected) of the parallel $R L C$ zero-sequence circuit defined as

$$
\omega_{0}=\frac{1}{\sqrt{L_{0} C_{0}}} .
$$

If the ideal resonant frequency $\omega_{0}$ is equal to the system fundamental frequency $\omega$, the circuit is tuned $(\nu=0)$, which means that the earth-fault capacitive current is completely compensated by the inductive coil current. The remaining earth-fault current is determined by the dumping $d$.

The unbalance factor

$$
k_{n}=\sqrt{d^{2}+\nu^{2}} \frac{U_{n}}{U}
$$

is yet another important parameter that describes operation of the compensated networks. The typical range of values for the unbalance factor is between 0.001 and 0.03 . $U_{n}$ and $U$ are neutral-to-ground voltage and a phase-to-neutral voltage, respectively. In the case of full compensation $(\nu=0)$, according to (7), the voltage $U_{n}$ is proportional to the voltage $U$ with the proportionality factor $k_{n} / d$. If most of the lines are overhead lines (sometimes untransposed) then, for the typical range of damping values $(d=0.01 \div 0.05)$, we can expect prohibitively large values for the neutral voltage $U_{n}$. To limit the neutral voltage $U_{n}$, it is common to have overcompensation $(\nu<0)$ in the system. Undercompensation $(\nu>0)$ could lead to the case of full compensation if some lines are disconnected, and it is not used in practice.

\section{PARAMETER IDENTIFICATION TECHNIQUE}

\section{A. Transients During the Recovery Process}

The starting point in time for the analysis of the recovery process is the moment when arc disappears. The phase voltage returns back to the normal operating value and, at the same time, the neutral voltage reduces to the small value proportional to the system unsymmetry. This transient process has been modeled using the superposition circuit with the current source equal to the negative value of the earth-fault current (3)

$$
i_{e}(t)=\sqrt{d^{2}+\nu^{2}} I_{c} \sin \omega t
$$

The recovery voltage is calculated using the following expression:

$$
u(t)=\mathcal{L}^{-1}\left\{\frac{1}{3} Z_{0}(s) I_{e}(s)\right\}
$$

where $\mathcal{L}^{-1}\{\bullet\}$ is the inverse Laplace transform, $Z_{0}(s)$ is the zero-sequence impedance (1) in the $s$-domain, and $I_{e}(s)$ is the Laplace Transform of the earth-fault current (8). The $\alpha$-component impedance has a negligibly small value compared to the zero-component impedance and it is omitted in (9). According to (1), the expression for the zero-component impedance in the $s$-domain is

$$
Z_{0}(s)=\frac{1}{C_{0}} \frac{s}{s^{2}+2 \delta s+\delta^{2}+\tilde{\omega}^{2}}
$$

where

$$
\delta=\frac{\omega d}{2}
$$

and

$$
\tilde{\omega}=\sqrt{\omega_{0}^{2}-\delta^{2}}
$$

The parameters $d$ and $\omega_{0}$ are defined in (4) and (6), respectively.

By incorporating (10) and the Laplace Transform of (8) in (9), and representing the capacitive earth-fault current using the prefault voltage (i.e., $I_{c}=3 \omega C_{0} U_{p f}$ ), we obtained the following expression for the recovery voltage:

$$
\begin{aligned}
u(t)= & U_{p f} \sqrt{(2 \delta \omega)^{2}+\left(\omega^{2}-\tilde{\omega}^{2}-\delta^{2}\right)^{2}} \\
& \times \mathcal{L}^{-1}\left\{\frac{s}{(s+j \omega)(s-j \omega)(s+\delta+j \tilde{\omega})(s+\delta-j \tilde{\omega})}\right\} .
\end{aligned}
$$

According to (13), the recovery voltage consists of two sinusoidal components

$$
u(t)=A_{1} \cos \left(\omega t+\varphi_{1}\right)-A_{2} e^{-\delta t} \cos \left(\tilde{\omega} t+\varphi_{2}\right) .
$$

In addition, the neutral-to-ground voltage during the recovery process could be calculated using the superposition theorem. On the phase recovery voltage (14), we add the neutral voltage during the earth-fault condition before the moment when arc extinguishes. Taking into consideration unsymmetry in the network, the expression for the neutral voltage will have the same form as (14) with a much smaller amplitude $A_{1}$.

The resonant-earthed system operating parameters (4) and (5) could be calculated if $\delta$ and $\widetilde{\omega}$ are known. It is possible to estimate $\delta$ and $\widetilde{\omega}$ by matching the recorded neutral voltage with the model (14). The estimation technique is presented in the following section. 


\section{B. Parameter Estimation}

According to the analysis in Section III-A, the neutral voltage signal consists of the fundamental frequency sinusoid and one damped sinusoid with the resonant frequency that is very close to the fundamental frequency. In practice, it is possible to have other components in the signal, predominantly the odd number harmonics $(3 \mathrm{rd}, 5 \mathrm{th}, 7 \mathrm{th}, \ldots)$ of both, the fundamental frequency and the resonant frequency. Since the resonant frequency sinusoid is damped, the corresponding harmonics will be also damped. These harmonics in the neutral voltage are generated from the nonlinearities in the zero-sequence circuit. The odd harmonics indicate the presence of saturation nonlinearity (neutral coil saturation for example). Sometimes constant even harmonics (e.g., 6th), could be seen on the spectrogram of the recorded neutral voltage. These harmonics are always zero sequence, and they come from other nonlinearities in the network.

During the earth fault condition, the voltages of unaffected phases increase to the values equal to the prefault phase-tophase voltage and the neutral voltage increases to the prefault phase voltage value. Higher voltages in the network will make nonlinearities more critical. This effect is clearly observable in the fault records [5]. The amplitudes of the current harmonics are very high comparing to the fundamental frequency. It is important to understand that for the tuned system, the capacitive component of the $k$ th harmonic is exactly $k^{2}$ larger then the inductive component. Hence, the compensation does not have any effect on harmonics and it is possible for the current harmonics to have larger amplitudes then the fundamental frequency current [5]. When arc is extinguished, the major source of odd harmonics disappears but still the effect of other saturation nonlinearities could be seen in the records. If the resonant frequency $\widetilde{\omega}$ is different then the fundamental frequency $\omega$, the amplitude of the phase recovery voltage will oscillate slowly with the frequency $(\widetilde{\omega}-\omega) / 2$. In this oscillation, the voltage could reach values of up to $70 \%$ above the rated value, and arc could establish and extinguish in short periods when the phase recovery voltage reaches maximum values. The conclusion of this analysis would be that we should expect in the recorded signal: constant and time-varying odd harmonics of fundamental frequency and resonant frequency, respectively, and sometimes constant zero-sequence even harmonics.

In the first part of the parameter estimation algorithm, the troublesome signal components are filtered out using the lowpass linear-phase (FIR) filter with the cutoff frequency $130 \mathrm{~Hz}$. Also, the fundamental frequency has been removed by convoluting the signal with the following notch filter:

$$
B(z)=\prod_{k=1}^{n}\left(1-e^{\underline{z}_{k}} z^{-1}\right)=1+\sum_{k=1}^{n} b_{k} z^{-k}
$$

where in our case $n=2$ and $\underline{z}_{k}$ are complex conjugate poles representing the fundamental frequency.

The filtered signal represents only the transient component of the measured neutral voltage and it has the following model:

$$
u(q)=\bar{u}(q)-\Delta u(q)
$$

where

$$
\bar{u}(q)=A_{2} e^{-\delta T_{s} q} \cos \left(\tilde{\omega} T_{s} q+\varphi_{2}\right)
$$

where $q$ is the sample number, $T_{s}$ is the sampling interval, and $\Delta u(q)$ represents measurement error. It is assumed that the measurement errors are independent and identically distributed random numbers (normal distribution). The error-free signal (17) satisfies exactly the following difference equation:

$$
\bar{u}(q)+x_{1} \bar{u}(q-1)+x_{2} \bar{u}(q-2)=0
$$

where

$$
x_{1}=-2 e^{-\delta T_{s}} \cos \tilde{\omega} T_{s}
$$

and

$$
x_{2}=e^{-2 \delta T_{s}} \text {. }
$$

In the difference equation (18), we replaced the error-free signal (17) with the measured signal plus error according to (16). The difference equation is satisfied for each sample in data window spanning $m$ samples, that is

$$
\begin{aligned}
& u(q)+\Delta u(q)+x_{1}[u(q-1)+\Delta u(q-1)] \\
& \quad+x_{2}[u(q-2)+\Delta u(q-2)]=0, \quad q=3,4, \ldots, m
\end{aligned}
$$

or in the matrix notation

$$
\mathbf{A x}+\mathbf{b}+\mathbf{D}(\mathbf{x}) \Delta \mathbf{u}=0
$$

where

$$
\begin{aligned}
& \mathbf{x}^{T}=\left[x_{1} x_{2}\right], \quad \mathbf{x} \in \mathbf{R}^{2 \times 1} \\
& \Delta \mathbf{u}^{T}=[\Delta u(1) \Delta u(2) \ldots \Delta u(m)], \quad \Delta \mathbf{u} \in \mathbf{R}^{m \times 1} \\
& \mathbf{A}^{T}=\left[\begin{array}{llll}
u(2) & u(3) & \ldots & u(m-1) \\
u(1) & u(2) & \ldots & u(m-2)
\end{array}\right], \quad \mathbf{A} \in \mathbf{R}^{(m-2) \times 2} \\
& \mathbf{b}^{T}=[u(3) u(4) \ldots u(m)], \quad \mathbf{b} \in \mathbf{R}^{(m-2) \times 1} \\
& \mathbf{D}(\mathbf{x})=\left[\begin{array}{cccccc}
x_{2} & x_{1} & 1 & 0 & \ldots & 0 \\
0 & x_{2} & x_{1} & 1 & \ddots & \vdots \\
\vdots & \ddots & \ddots & \ddots & \ddots & 0 \\
0 & \ldots & 0 & x_{2} & x_{1} & 1
\end{array}\right] \\
& \mathbf{D}(\mathbf{x}) \in \mathbf{R}^{(m-2) \times m} \text {. }
\end{aligned}
$$

According to (22), the error vector can be written as

$$
\Delta \mathbf{u}=-\mathbf{D}(\mathbf{x})^{+}(\mathbf{A x}+\mathbf{b})
$$

where "+" indicates the matrix pseudoinversion. The optimal estimates of the parameters $x_{1}$ and $x_{2}$ correspond to the minimum of the norm $\Delta \mathbf{u}^{T} \Delta \mathbf{u}$. The estimation problem is nonlinear and can be formulated as IRLS problem [8]

$$
\min _{\mathbf{x}}(\mathbf{A} \mathbf{x}+\mathbf{b})^{T} \mathbf{W}(\mathbf{x})(\mathbf{A} \mathbf{x}+\mathbf{b})
$$

where $\mathbf{W}(\mathbf{x})=\left[\mathbf{D}(\mathbf{x}) \mathbf{D}(\mathbf{x})^{T}\right]^{-1}$ is the weighting matrix. An iterative algorithm for solving the IRLS problem is provided in [8]. 
Using (19), (20), (11), and (12), we derived the following formulas to calculate the compensated network parameters from the estimates $x_{1}$ and $x_{2}$ :

$$
d=-\frac{\ln x_{2}}{\omega T_{s}}
$$

and

$$
\omega_{0}=\frac{1}{T_{s}} \times \sqrt{\left(\frac{\ln x_{2}}{2}\right)^{2}+\left(\cos ^{-1} \frac{-x_{1}}{2 \sqrt{x_{2}}}\right)^{2}} .
$$

Detuning parameter (5) is calculated using the estimate of the frequency (26).

\section{Simulation Study}

The goal of the simulation study is to compare the proposed IRLS signal estimation technique (24) with the classical linear least-squares (LS) estimation $\hat{\mathbf{x}}=\mathbf{A}^{+} \mathbf{b}$, where "+" indicates matrix pseudoinversion. $\mathbf{A}$ and $\mathbf{b}$ are defined as in (22). It should be noted that the LS solution is obtained from the simplified error model. A difference (21) for the sample $q$ includes the error $\Delta u(q)$ but assumes $\Delta u(q-1)=\Delta u(q-2)=0$.

The following signal represents the neutral-to-ground voltage during a recovery process (with fundamental frequency and harmonics filtered-out):

$$
u(q)=30 e^{-\delta q T_{s}} \cos \left(\widetilde{\omega} q T_{s}\right)+\Delta u(q) .
$$

The signal is sampled with 200 samples/s sampling frequency to speed up the simulation. The signal $u(q)$ amplitude and the standard deviation $\sigma$ of the measurement error $\Delta u(q)$ are values relative to the fundamental frequency amplitude. The parameter $\delta$ is calculated using (11) for the specified damping $d=0.05$. The system is overcompensated with the detuning factor $\nu=$ -0.0404 (corresponds to 51-Hz ideal resonant frequency of the lossless resonant circuit). The resonant frequency $\widetilde{\omega}$ is calculated according to (12). The standard deviation $\sigma$ of the measurement error $\Delta u(q)$ has been increased from 0.005 to 0.105 with the steps 0.01 . For each error level, 100 runs of the IRLS estimator (24) have been conducted with the errors simulated using the random number generator. Also, the classical LS solution has been calculated for each run. In fact, the LS solution is used to start an iterative IRLS solver. Absolute errors in damping and detuning estimation are calculated in each estimator run and averaged to find the asymptotic mean values. Simulation results are presented in Fig. 2. These results clearly show the superiority of the IRLS estimator in comparison to the classical LS estimator. The IRLS is ten times more accurate then the LS method.

The solution method of the IRLS problem (24) is iterative. The simulation study has revealed that the average number of iterations (averaged over 100 simulation runs) is constant for moderate noise levels and it will increase linearly for higher noise levels, as shown in Fig. 3. The objective function of the problem (24) is nonlinear and it has many local minimums. The starting values in the iterative method are very critical. We have been using the values that are the solution to the LS problem. It should be noticed that for high noise levels, the LS solution is moving apart from the optimal solution of the problem (24).

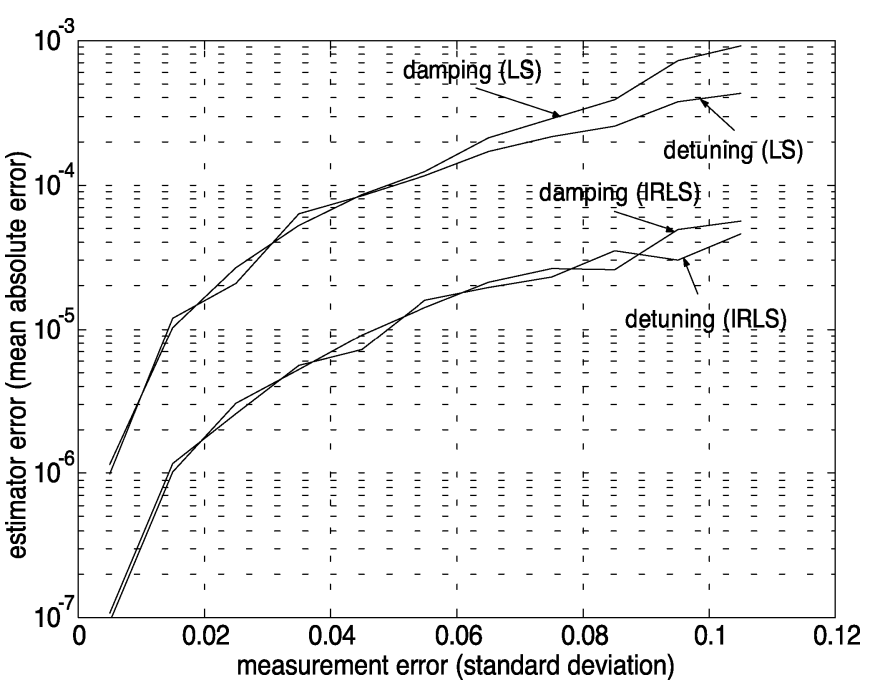

Fig. 2. Mean absolute error in damping and detuning estimation as a function of measurement error standard deviation for IRLS and LS methods.

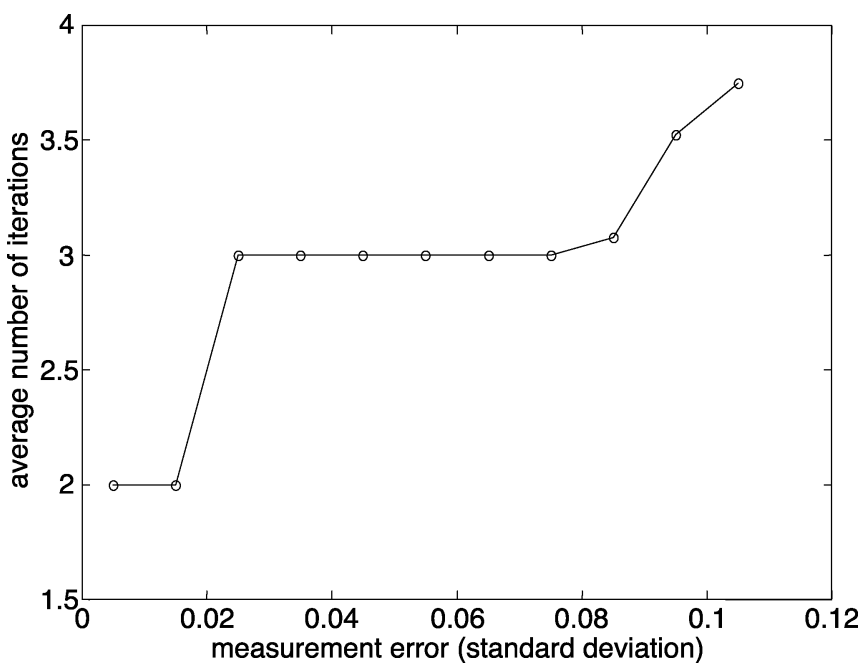

Fig. 3. Average number of iterations for IRLS method as a function of measurement error.

Hence, we can expect an increase in the number of iterations and possible convergence to a local minimum. However, in this application we never encountered this problem.

\section{Effects of Prefiltering}

There are two filters before the IRLS algorithm: the lowpass filter with the cutoff frequency $130 \mathrm{~Hz}$, and the notch filter to remove the fundamental frequency $(50 \mathrm{~Hz})$. The effects of filtering are the attenuation of both the amplitude $A_{2}$ and the noise in the filtered signal (16). The parameter $A_{2}$ is only the scaling factor in the model (21) and it is not estimated. The ratio between the amplitude attenuation and the noise attenuation controls the actual signal-to-noise ratio (SNR) in the model (21). In the FIR filters, the output noise standard deviation is obtained by multiplying the input noise standard deviation with the reduction factor $\sqrt{\sum_{k=0}^{n} b_{k}^{2}}$, where $b_{k}$ are filter coefficients. The reduction factor of the low-pass filter is 0.37 , while for the notch filter, it is 1.41 (for the sampling frequency 200 samples/s). The transfer function of the notch filter is $H(\widetilde{\omega})=$ 

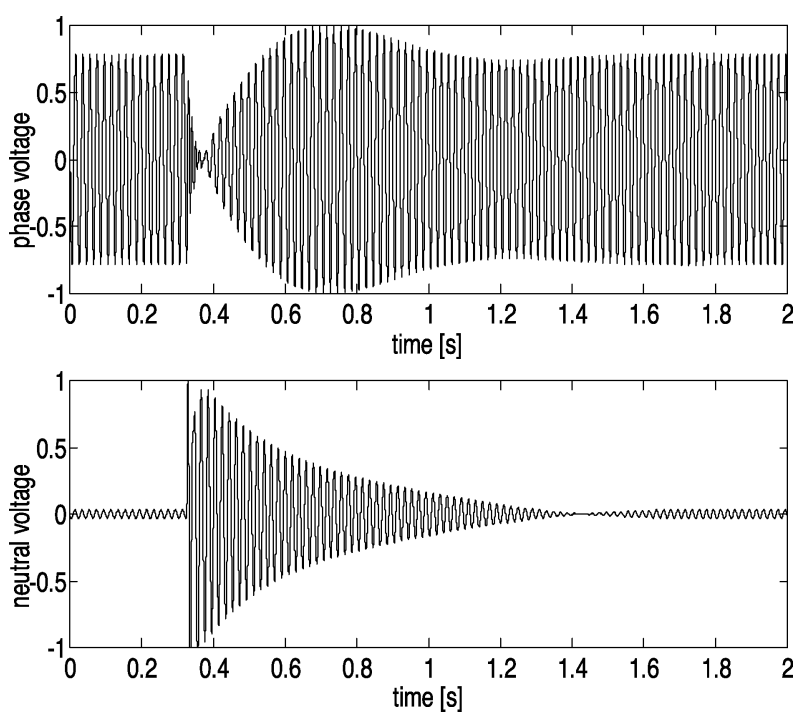

Fig. 4. Faulty phase voltage and neutral voltage transients (normalized values) during the staged earth-fault.

$2 \cos \left(\widetilde{\omega} T_{s}\right)-2 \cos \left(\omega T_{s}\right)$, where $\omega$ and $T_{s}$ are the fundamental frequency and the sampling interval, respectively. According to this transfer function, the attenuation of $A_{2}$ will increase as the resonant frequency approaches the fundamental frequency. The passband ripple of the low-pass filter is completely insignificant in comparison to the attenuation effect of the notch filter. We can conclude from this analysis that the prefiltering lowers the SNR in the model (21) as the resonant frequency comes closer to the fundamental frequency. This result justifies the use of IRLS instead of LS to fit the model (21).

\section{Practical Results}

The staged earth-fault records [5] are used to test the practical value of the identification method proposed in Section III. These records are obtained in the 110-kV network of one German utility. Details about the network and the measurement layout are presented in [5]. We selected to present here the result obtained for the fault record shown in Fig. 4. The neutral-to-ground voltage signal (lower part of Fig. 4) has been selected as an input to the IRLS method (24). The analysis of this signal reviled the presence of odd harmonics starting with the 3rd of both fundamental and resonant frequencies. To illustrate this phenomenon, the relative amplitudes of the resonant frequency-3rd and 5 th harmonics (of the resonant frequency) are obtained by using windowed DFT. The results are shown in Fig. 5. It should be noticed that the resolution of DFT is not good enough to make difference between the fundamental frequency and the resonant frequency. Hence, in Fig. 5, the constant values before and after the transient part (in the middle) represent the fundamental frequency and its harmonics. During the fault, harmonics increase sharply as could be seen in Fig. 5. As suggested in Section III-B, it is very important for this analysis to apply the low-pass filter before using the IRLS estimation method. The parameters identified in this example are the damping $d=0.0206$ and the detuning $\nu=-0.0423$.

The simulation study results reported in Section III-C indicate that the IRLS method is significantly more accurate than the LS

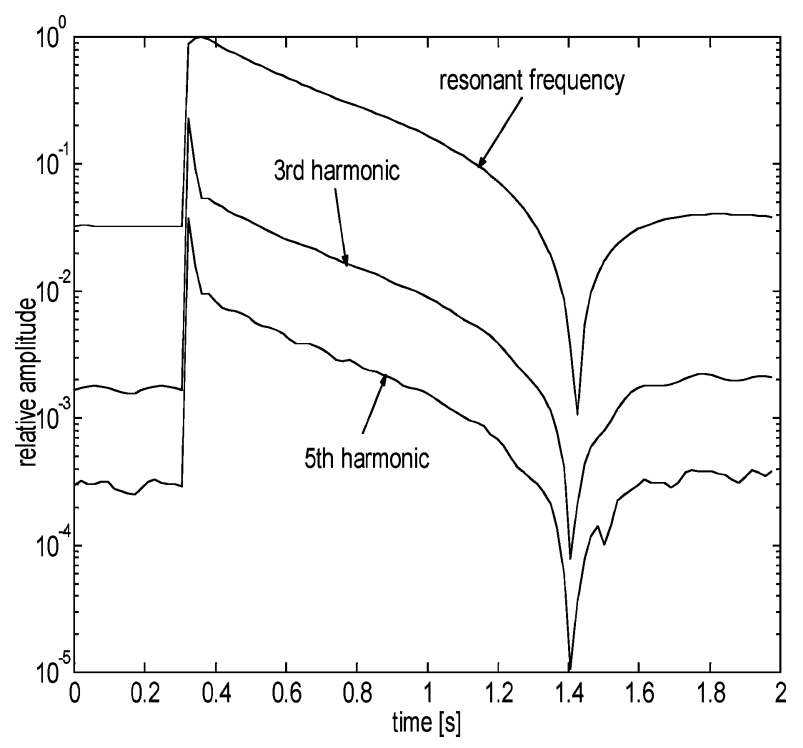

Fig. 5. Relative amplitudes of the frequency components in the neutral-to-ground voltage during the staged earth-fault.

TABLE I

COMPARISON OF IRLS AND LS ESTIMATES

(REC. IS THE ABBREVIATION FOR DATA RECORD AND DIFF. IS THE ABBREVIATION FOR DIFFERENCE)

\begin{tabular}{c|l|l|c|c|c|c}
\hline \multicolumn{2}{c|}{} & $\omega_{0}$ & $d$ & $v$ & $x_{1}$ & $x_{2}$ \\
\hline \multirow{2}{*}{$\begin{array}{c}\text { rec. } \\
1\end{array}$} & IRLS & 50.544 & .0216 & -.0219 & .0333 & .9666 \\
\cline { 2 - 7 } & LS & 49.91 & .0633 & .0036 & -.0079 & .9054 \\
\cline { 2 - 7 } & diff. & $1.25 \%$ & $193 \%$ & $116 \%$ & $124 \%$ & $6.33 \%$ \\
\hline \multirow{2}{*}{ rec. } & IRLS & 50.761 & .0204 & -.0307 & .0468 & .9685 \\
\cline { 2 - 7 } 2 & LS & 50.722 & .0262 & -.0291 & .044 & .9597 \\
\cline { 2 - 7 } & diff. & $0.077 \%$ & $28 \%$ & $5.21 \%$ & $5.98 \%$ & $0.91 \%$ \\
\hline \multirow{2}{*}{$\begin{array}{c}\text { rec. } \\
3\end{array}$} & IRLS & 51.078 & .0207 & -.0436 & .0664 & .9681 \\
\cline { 2 - 7 } & LS & 50.687 & .03 & -.0277 & .0416 & .954 \\
\cline { 2 - 7 } & diff. & $0.77 \%$ & $45 \%$ & $36.5 \%$ & $38.5 \%$ & $1.46 \%$ \\
\hline \multirow{2}{*}{ rec. } & IRLS & 51.369 & .0217 & -.0555 & .0843 & .9665 \\
\cline { 2 - 7 } 4 & LS & 51.389 & .023 & -.0563 & .0854 & .9646 \\
\cline { 2 - 7 } & diff. & $0.038 \%$ & $6 \%$ & $1.44 \%$ & $1.3 \%$ & $0.2 \%$ \\
\hline
\end{tabular}

TABLE II

Prediction MEAN ABSOLUTE ERROR FOR IRLS AND LS METHODS

\begin{tabular}{c|l|l|l|l|l|l|l}
\hline \multicolumn{2}{c|}{ record 1 } & \multicolumn{2}{c|}{ record 2 } & \multicolumn{2}{c|}{ record 3 } & \multicolumn{2}{c}{ record 4 } \\
\hline IRLS & LS & IRLS & LS & IRLS & LS & IRLS & LS \\
\hline .1236 & .246 & .0585 & .101 & .0335 & .056 & .1021 & .13 \\
\hline
\end{tabular}

method. We present here the identification results for four representative earth-fault records. These results are substantiating the simulation results and further emphasizing the importance of using IRLS instead of LS method in this application. The results are tabulated in Tables I and II. The identification results for the following parameters are compared (Table I):

$\omega_{0} \quad$ ideal resonant frequency defined in (6);

$d \quad$ damping defined in (4) and estimated using (25);

$\nu \quad$ detuning defined in (5) and estimated using (26);

$\left(x_{1}, x_{2}\right) \quad$ solutions of the IRLS problem (24) and the simplified LS problem.

The percentage difference (abbreviated as diff.) in Table I is calculated as an absolute difference between IRLS and LS solutions divided by the IRLS solution and multiplied by 100 . The 
results presented in Table I denote a considerable difference between IRLS and LS solutions. The IRLS and LS solutions are verified by comparing predicted and recorded signals. The index used in this comparison is the prediction mean absolute error, defined as a mean of absolute difference between predicted and recorded signals. The results of this verification test (Table II) indicate that the parameter estimates obtained by using the IRLS method are much closer to the actual values than the LS estimates.

\section{CONCLUSION}

It has been shown in this paper that the records of transient faults could be used to identify damping and detuning parameters in the resonantly grounded systems. When arc disappears, the neutral-to-ground voltage returns back to the prefault value. The model of the neutral voltage signal is parameterized according to the known mathematical model of this transient process. Damping and resonant frequency (detuning) are the key parameters in this model. To fit the model, the IRLS method has been used. The simulation study has been conducted to compare the IRLS with the classical least squares method. The results clearly indicate the superior quality of the IRLS method. The method could be completely disrupted if harmonics are present in the signal. Therefore, use of the low-pass filter has been suggested to mitigate the problem. This algorithm has been used to analyze the records of some staged faults in one German utility. Illustrative practical examples are presented in this paper.

\section{REFERENCES}

[1] W. Petersen, "Limitation of earth current and suppression of earth fault arcs by the earthing coil," ETZ Elektrotechnische Zeitschrift, vol. 40, 1919.

[2] IEEE Recommended Practice for Grounding of Industrial and Commercial Power Systems, IEEE Std. 142-1991, 1992.

[3] V. Leitloff, L. Pierrat, and R. Feuillet, "Study of the neutral-to-ground voltage in a compensated power system," Eur. Trans. Elect. Power Eng. (ETEP), vol. 4, no. 2, pp. 145-153, Mar./Apr. 1994.

[4] D. Griffel, V. Leitloff, Y. Harmand, and J. Bergeal, "A new deal for safety and quality on MV networks," IEEE Trans. Power Delivery, vol. 12, pp. 1428-1433, Oct. 1997.
[5] P. Martel, H. J. Radtke, P. Schegner, and O. Seifert, "Results of earth fault measurements in an earth fault compensated $110 \mathrm{kV}$ system," in Proc. 16th Int. Conf. Electricity Distribution (CIRED), Amsterdam, The Netherlands, 2001

[6] O. Chaari, P. Bastard, and M. Meunier, "Prony's method: An efficient tool for the analysis of earth fault currents in Petersen-coil-protected networks," IEEE Trans. Power Delivery, vol. 10, pp. 1234-1241, July 1995.

[7] P. Schegner, G. Pilz, and O. Seifert, "Methods for automatic parameter estimation of resonant-earthed systems," in Proc. Modern Electric Power Systems, Wroclaw, Poland, Sept. 2002.

[8] A. Bjork, Numerical Methods for Least Squares Problems. London, U.K.: SIAM, 1996, p. 173.

Rastko Živanović (M'97) received the Dipl.Ing. and M.Sc. degrees from the University of Belgrade, Belgrade, Serbia, in 1987 and 1991, and the Ph.D. degree from the University of Cape Town, Cape Town, South Africa, in 1997.

Currently, he is Professor with the Faculty of Engineering at Tshwane University of Technology, Pretoria, South Africa, where he has also been Lecturer and Senior Lecturer since 1992. His research interests include power system protection and control

Peter Schegner (M'99) received the M.Sc. degree in electrical power engineering from the Darmstadt University of Technology, Darmstadt, Germany, in 1982, and the Ph.D. degree from Saarland University, Saarland, Germany, in 1989.

Currently, he is Full Professor and Head of the Institute of Electrical Power Systems and High Voltage Engineering, Dresden University of Technology, Dresden, Germany. He was a System Engineer in the field of power system control and became a member of the scientific staff at the Saarland University. From 1989 to 1995, he was Head of the Development Department of Protection Systems at AEG, Frankfurt A.M., Germany.

Olaf Seifert received the M.Sc. degree in electrical power engineering from the Dresden University of Technology, Dresden, Germany, where he is currently pursuing the $\mathrm{Ph} . \mathrm{D}$. degree.

$\mathrm{He}$ is a Member of the Scientific Staff in the Institute of Electrical Power Systems, Dresden University of Technology.

Georg Pilz received the M.Sc. degree in electrical power engineering from the Dresden University of Technology, Dresden, Germany, where he is currently pursuing the Ph.D. degree.

$\mathrm{He}$ is a Member of the Scientific Staff in the Institute of Electrical Power Systems, Dresden University of Technology. 